\title{
Proteoglycans Regulate the Chemotaxis of Dendritic Cells Derived from Human Peripheral Blood Monocytes
}

\author{
Hironori Yoshino, Kenji TaKahashi, Satoru Monzen, and Ikuo KashiwaKura* \\ Department of Radiological Life Sciences, Division of Medical Life Sciences, Hirosaki University Graduate School of \\ Health Sciences; 66-1 Hon-cho, Hirosaki, Aomori 036-8564, Japan. \\ Received December 17, 2009; accepted March 11, 2010
}

\begin{abstract}
Dendritic cells (DCs) are a type of antigen-presenting cell which play an essential role in the immune system. The transition from immature DC (iDCs) to mature DCs (mDCs) requires appropriate maturation stimuli, such as pro-inflammatory cytokines or pathogen-derived components. Proteoglycans (PGs), which are composed of core proteins and the glycosaminoglycans that bind to them, are one of the main components of the extracellular matrix around pathogens such as bacteria. This study investigated the effects of PG extracted from the nasal septum cartilage of whale (W-PG) on the maturation of DCs derived from human peripheral blood monocytes. iDCs were prepared from human monocytes using granulocyte-macrophage colony-stimulating factor (GM-CSF) and interleukin-4 (IL-4). The iDCs were stimulated by W-PG alone. In another type of experiment, the iDCs were stimulated by MIX (tumor necrosis factor- $\alpha$ (TNF- $\alpha$ ), IL-1 $\beta$, IL-6 and prostaglandin $\mathrm{E}_{2}\left(\mathrm{PGE}_{2}\right)$ ) or a combination of MIX plus W-PG. The stimulation of W-PG alone did not induce the phenotypic maturation from iDCs. However, W-PG promoted the up-regulation of chemokine receptor CCR7-surface expression and the chemotactic responsiveness to CCR7 ligand macrophage inflammatory protein-3 $\beta$ on MIX-stimulated mDCs although WPG did not influence matrix metalloproteinase-9 activity which is an important factor in DC migration through the extracellular matrix. The findings that W-PG can selectively regulate the chemotactic activity of DCs in vitro under inflammatory conditions therefore indicate that the interaction of PGs with immune cells including DCs plays an important role in the immune response under the milieu of innate immunity.
\end{abstract}

Key words dendritic cell; proteoglycan; CC chemokine receptor 7; chemotaxis

Dendritic cells (DCs) play key roles in immune regulation as professional antigen-presenting cells. There are three stages of differentiation: DC precursors, immature DCs (iDCs), and mature DCs (mDCs). ${ }^{1-3)}$ iDCs locate in various tissues and they migrate to inflammatory sites in response to chemokines such as CC-chemokine ligand CCL5. At inflammatory sites, iDCs capture antigens such as invading bacteria and viruses and then process the antigens for presentation on major histocompatibility complex molecules. Pathogen-derived components or pro-inflammatory cytokines can induce the DC maturation. During maturation, the DCs express CCchemokine receptor CCR7 and migrate from the inflammation site to the lymphoid organs in response to chemokines such as CCL19 (macrophage inflammatory protein-3 $\beta$ : MIP$3 \beta$ ) and CCL21. ${ }^{4)}$ mDCs acquire a high antigen-presenting capacity instead of losing their phagocytotic activity and thereby vigorously stimulate the T-cell responses. ${ }^{2)}$

The extracellular matrix and glycosaminoglycans (GAGs) actively contribute to a variety of physiological processes, including the development of an immune response. ${ }^{5)}$ GAGs such as hyaluronan and chondroitin sulfate A can act as immunomodulators by activating DCs under certain conditions. ${ }^{5,6)}$ Proteoglycans (PGs), which are composed of core proteins and the GAGs that bind to them, are one of the main components of the extracellular matrix around pathogens such as bacteria. PGs also can modulate immune responses as well as GAGs. For example, some previous studies reported that PGs purified from Agaricus blazei and Phellinus linteus could activate immune cells and induce anti-tumor effects. $^{7-9)}$ Furthermore, these PGs can induce maturation of DCs derived from murine bone marrow. ${ }^{7-9)}$

Majima et al. succeeded in extracting PG from the nasal cartilage of the salmon, which had only been considered waste material. ${ }^{10)}$ The potential benefits of salmon PG have been widely investigated, and previous reports have described the biological activities of salmon PG. ${ }^{11,12)}$ Although the effect of salmon PG on human immune cells was investigated, it has no effect on the maturation of human monocytederived DCs. ${ }^{13)}$ Our recent work has demonstrated that PGs extracted from the nasal septum cartilage of whale (W-PG) promote the generation of megakaryocytic progenitor cells and megakaryocytes in culture with a lower dose of cytokines, while salmon PG had no promoting effect. ${ }^{14)}$ These results indicate that the effect of $\mathrm{PG}$ depends on the species. Therefore, the effect of W-PG on human immune cells may be different from that of salmon PG. However, the effects of W-PG on human immune cells have not yet been elucidated. Specifically, the effects of W-PG on the maturation of DCs have not been investigated. This study therefore, investigated the effects of W-PG on the maturation of DCs derived from human peripheral blood monocytes.

\section{MATERIALS AND METHODS}

Reagents Recombinant human granulocyte-macrophage colony-stimulating factor (rhGM-CSF), interleukin-1 $\beta$ (rhIL$1 \beta$ ), rhIL-4, rhIL-6, tumor necrosis factor- $\alpha$ (rhTNF- $\alpha$ ), and rhMIP-3 $\beta$ were purchased from PeproTech Inc. (Rocky Hill, NJ, U.S.A.). Prostaglandin $\mathrm{E}_{2}\left(\mathrm{PGE}_{2}\right)$ was purchased from Sigma-Aldrich, Inc. (Tokyo, Japan). Fluorescence-labeled monoclonal antibodies (mAbs), anti-human cluster of differentiation 1a-phycoerythrin (CD1a-PE), CD2-fluorescein isothiocyanate (CD2-FITC), CD40-FITC, human leukocyte antigen-ABC-FITC (HLA-ABC-FITC), CCR5-FITC, unconjugated CCR7, mouse immunoglobulin $\mathrm{G}_{2 \mathrm{a}}\left(\mathrm{IgG}_{2 \mathrm{a}}\right)-\mathrm{PE}$, and rat $\mathrm{IgG}_{2 \mathrm{a}}-\mathrm{PE}$ were purchased from Becton Dickenson (San 
Jose, CA, U.S.A.). Anti-human CD8-FITC, CD19-FITC, CD80-FITC, CD4-PE, CD11c-PE，CD14-PE，CD83-PE, CD86-PE, mouse IgG $_{1}$-FITC and mouse IgG $_{2 b}$-PE were purchased from Beckman Coulter (Fullerton, CA, U.S.A.) and anti-human CD3-PE and human leukocyte antigen (HLA)DR-PE were obtained from eBioscience (San Diego, CA, U.S.A.). FITC-conjugated goat anti-mouse IgM antibody was purchased from Santa Cruz Biotechnology (CA, U.S.A.).

Preparation and Characteristics of W-PG W-PG was prepared from the nasal septum cartilage of a Bryde whale (Balaenoptera edeni). These cartilages were purchased from The Institute of Cetacean Research (Tokyo, Japan), which is a whale research program approved by the International Convention for the Regulation of Whaling. The W-PG was extracted by the guanidine- $\mathrm{HCl}$ technique according to a method described in a previous report. ${ }^{15)}$ Each purified PG was dissolved in $\mathrm{Ca}^{2+}$ - and $\mathrm{Mg}^{2+}$-free phosphate-buffered saline (PBS). The GAG composition of W-PG was previously reported elsewhere. ${ }^{14)}$

The endotoxin level of W-PG was tested using Limulus Amebocyte Lysate PYROGENT ${ }^{\circledR}$ Single Test Vials (Cambrex Bio Science Walkersville, Inc. Walkersville, MD, U.S.A.). The maximum concentration of W-PG used in this study was $100 \mu \mathrm{g} / \mathrm{ml}$ and $100 \mu \mathrm{g} / \mathrm{ml} \mathrm{W-PGs} \mathrm{contained} \mathrm{less}$ than $10 \mathrm{pg} / \mathrm{ml}$ endotoxin. This endotoxin level is the same level as the endotoxin contained in $50 \mathrm{ng} / \mathrm{ml} \mathrm{rhGM-CSF}$ plus $50 \mathrm{ng} / \mathrm{ml} \mathrm{rhIL}-4$.

Collection of Monocytes from Buffy-Coat This study was approved by the Committee of Medical Ethics of Hirosaki University School of Medicine (Hirosaki, Japan). After obtaining informed consent from all of the normal human blood donors, peripheral blood was collected by the Aomori Red Cross Blood Center. The peripheral blood mononuclear cells (PBMCs) were separated from the buffycoat $(n=32)$ by centrifugation with Lymphosepar I (1.077 $\mathrm{g} / \mathrm{ml}$; Immuno-Biological Laboratories Co., Ltd., Takasaki, Japan). The PBMCs were collected and washed at least three times with PBS containing $5 \mathrm{~mm}$ ethylenediaminetetraacetic acid (EDTA). The monocytes were separated from the PBMCs as described previously ${ }^{16)}$ with some modification. In brief, when the PBMCs contained more than $2.0 \times 10^{8}$ cells, the monocytes were separated from the PBMCs by centrifugation for $15 \mathrm{~min}$ at $580 \times \boldsymbol{g}$ on a cushion of a hyper-osmotic percoll solution. ${ }^{17)}$ After centrifugation, the monocyte fractions were collected and washed with PBS containing $5 \mathrm{~mm}$ EDTA. Thereafter, the number of these cells was counted. The purity of $\mathrm{CD}_{14}{ }^{+}$monocytes in these cells was $65.5 \pm 7.8 \%$ based on flow cytometry. In order to exclude all cells except monocytes from the cells, these cells were resuspended in PBS and allowed to adhere to a plastic dish (up to $5.0 \times 10^{6}$ cells per $60 \mathrm{~mm}$ dish) at $37^{\circ} \mathrm{C}$ for $1.5-2.0 \mathrm{~h}$ in a humidified atmosphere containing $5 \% \mathrm{CO}_{2}$. Thereafter, nonadherent cells were washed out with PBS. On the other hand, when the PBMCs contained less than $2.0 \times 10^{8}$ cells, the PBMCs were resuspended in PBS and allowed to adhere to a plastic dish (up to $2.0 \times 10^{7}$ cells per $60 \mathrm{~mm}$ dish) as described above. Thereafter, non-adherent cells were washed out with PBS. The adherent monocytes were cultured in RPMI 1640 (GIBCO, Grand Island, NY, U.S.A.) supplemented with $2 \%$ heat-inactivated human AB serum (GEMINI BIO-PRODUCTS, Woodland, CA, U.S.A.) and a $1 \%$ antibiotic-antimycotic mixture, which is hereinafter referred to as the medium.

In Vitro Generation and Culture of Human Dendritic Cells The DCs were generated from the monocytes according to previously published protocols ${ }^{18)}$ with some modification. In order to prepare the iDCs, the adherent monocytes were cultured in the presence of $50 \mathrm{ng} / \mathrm{ml} \mathrm{rhGM-CSF}$ plus $50 \mathrm{ng} / \mathrm{ml} \mathrm{rhIL}-4$ at $37^{\circ} \mathrm{C}$ for $5 \mathrm{~d}$ in a humidified atmosphere containing $5 \% \mathrm{CO}_{2}$. On day 3 during this 5-d culture, half of the culture medium was changed with new medium containing rhGM-CSF and rhIL-4 (50 ng/ml, respectively). After $5 \mathrm{~d}$ of culture, half of the medium was changed with new medium and the cells were re-cultured in the presence of $\mathrm{W}$ $\mathrm{PG}$ and/or maturation stimuli at $37^{\circ} \mathrm{C}$ in a humidified atmosphere containing $5 \% \mathrm{CO}_{2}$. In one type of experiment, the iDCs were stimulated by $100 \mu \mathrm{g} / \mathrm{ml} \mathrm{W}-\mathrm{PG}$ for $4 \mathrm{~d}$. After $4 \mathrm{~d}$ of culture, the cells were harvested for phenotypic analysis. In another type of experiment, the iDCs were stimulated by a cytokine mixture $(10 \mathrm{ng} / \mathrm{ml}$ rhTNF- $\alpha, 10 \mathrm{ng} / \mathrm{ml}$ rhIL- $1 \beta$, $10 \mathrm{ng} / \mathrm{ml} \mathrm{rhIL}-6$ and $1 \mu \mathrm{g} / \mathrm{ml} \mathrm{PGE}_{2}$ : MIX) or a combination of MIX+W-PG. A combination of MIX plus $100 \mu \mathrm{g} / \mathrm{ml}$ salmon PG was examined as previously reported. ${ }^{13)}$ After $2 \mathrm{~d}$ of culture, the cells were harvested for phenotypic and functional analysis and the supernatants were also collected and kept frozen at $-85^{\circ} \mathrm{C}$ to determine the cytokine concentration and perform a zymography assay.

Cell Surface Staining For the cell surface marker analysis of DCs, the induced cells were stained with mAbs conjugated to FITC or PE for $30 \mathrm{~min}$ at $4{ }^{\circ} \mathrm{C}$ in the dark. The cells were also stained with corresponding FITC- or PE-conjugated isotype control IgG. After $30 \mathrm{~min}$, the cells were washed with cold PBS and analyzed by flow cytometry (Epics XL, Beckman Coulter). For the CCR7 staining by a 2 step procedure, the cells were labeled with primary CCR7 antibodies for $20 \mathrm{~min}$ at $4{ }^{\circ} \mathrm{C}$ in the dark. Labeled cells were washed twice with PBS and stained with FITC-conjugated goat anti-mouse IgM secondary antibodies for $20 \mathrm{~min}$ at $4{ }^{\circ} \mathrm{C}$ in the dark. The induced cells from monocytes could be distinguished from the debris and lymphocytes using a region established by their high forward and side scatter signals as previously described. ${ }^{16)}$ The cell surface phenotypes of more than $6.0 \times 10^{3}$ cells within the region were analyzed. The data were expressed as the percentage of positive cells or mean fluorescence intensity (MFI).

Intracellular Staining of CCR7 The cells were suspended and fixed with Cytofix/Cytoperm ${ }^{\mathrm{TM}}$ solution (BD Bioscience, San Jose, CA, U.S.A.) for $20 \mathrm{~min}$ on ice. After washing twice with Perm/Wash ${ }^{\mathrm{TM}}$ solution (BD Bioscience), cells were suspended in Perm/Wash ${ }^{\mathrm{TM}}$ solution and labeled with primary CCR7 antibodies for $20 \mathrm{~min}$ at $4{ }^{\circ} \mathrm{C}$ in the dark. Labeled cells were washed twice with Perm/Wash ${ }^{\mathrm{TM}}$ solution and stained with FITC-conjugated goat anti-mouse IgM secondary antibodies for $20 \mathrm{~min}$ at $4{ }^{\circ} \mathrm{C}$ in the dark. As an isotype control, the cells were stained with FITC-conjugated goat anti-mouse IgM antibodies alone. After $20 \mathrm{~min}$, the cells were washed with Perm/Wash ${ }^{\mathrm{TM}}$ solution and analyzed by flow cytometry.

Chemotaxis Assay A cell migration assay was performed in a 24-well Transwell cell culture chamber (Costar, Corning, NY, U.S.A.) according to a previously published protocol $^{19,20)}$ with some modifications. In brief, the DCs 
$\left(1.0 \times 10^{5}\right.$ or $\left.2.0 \times 10^{5}\right)$ were re-suspended in a volume of $100 \mu$ l (RPMI 1640 supplemented with $2 \%$ heat-inactivated human $\mathrm{AB}$ serum) and then loaded in the upper chamber compartment. rhMIP-3 $\beta(100 \mathrm{ng} / \mathrm{ml})$ was added to the lower compartment in a volume of $500 \mu \mathrm{l}$ (RPMI 1640 supplemented with $2 \%$ heat-inactivated human $\mathrm{AB}$ serum). The lower compartment of the control chambers contained RPMI 1640 supplemented with $2 \%$ heat-inactivated human $A B$ serum alone. After $2 \mathrm{~h}$ of incubation at $37^{\circ} \mathrm{C}$ in a humidified atmosphere containing $5 \% \mathrm{CO}_{2}$, the cells that migrated through the $8 \mu \mathrm{m}$-pore size polycarbonate filters in the lower compartment were collected and counted by flow cytometry using Flow-Count ${ }^{\mathrm{TM}}$ Fluorospheres (Beckman Coulter). The percentage of migrated cells was calculated as: (the number of cells that migrated into the lower chamber containing MIP-3 $\beta$-number of cells that migrated in control medium)/ total number of cells loaded in the upper chamber $\times 100$.

Allogeneic Mixed Leukocyte Reaction Allogeneic $1.0 \times 10^{5} \mathrm{CD}^{+} \mathrm{T}$ cells $\left(95 \% \mathrm{CD}^{+} \mathrm{T}\right.$ cells $)$, which were purified from the PBMCs obtained from one individual using Human CD4 T lymphocyte Enrichment Set-DM (BD Bioscience), were co-cultured in 96-well flat bottom microplates (Asahi Techno Glass Co., Ltd., Chiba, Japan) with different numbers of DCs. Each stimulated DC was irradiated with 20 Gy using an X-ray generator (MBR-1520R, Hitachi Medical Corp., Tokyo, Japan) prior to co-culture with allogeneic $\mathrm{CD}^{+}{ }^{+} \mathrm{T}$ cells. Both cells were co-cultured for $3 \mathrm{~d}$ in RPMI 1640 supplemented with $2 \%$ heat-inactivated human $\mathrm{AB}$ serum at $37^{\circ} \mathrm{C}$ in a humidified atmosphere containing $5 \%$ $\mathrm{CO}_{2}$. After $3 \mathrm{~d}$ of culture, the cells were incubated for an additional $20 \mathrm{~h}$ in the presence of $\left[{ }^{3} \mathrm{H}\right]$-thymidine $(0.1 \mu \mathrm{Ci} /$ well, specific activity, $5 \mathrm{Ci} / \mathrm{mmol}$; Moravek Biochemicals Inc., CA, U.S.A.). To determine the proliferation of $\mathrm{CD}^{+}{ }^{+} \mathrm{T}$ cells, the cells were harvested onto glass fiber filters (Whatman, England) with a semiautomatic harvester (Labo Mash, Labo Science, Tokyo, Japan) and then the amount of $\left[{ }^{3} \mathrm{H}\right]$-thymidine incorporation was measured by a liquid scintillation counter (LSC-5100, Aloka Co., Ltd., Tokyo, Japan). As a negative control, $\mathrm{CD}^{+}{ }^{+} \mathrm{T}$ cells alone were cultured. The experiments were performed in triplicate cultures.

Cytokine Measurements The cytokines in the culture supernatants were measured using the Bio-Plex protein array system (Bio-Rad Laboratories, Hercules, CA, U.S.A.). The Bio-Plex cytokine assay is designed for the multiplexed quantitative measurement of multiple cytokines in a single well using as little as $50 \mu \mathrm{l}$ of sample. ${ }^{21)}$ For the cytokine assays, premixed multiplex beads of the Bio-Plex human cytokine 27-Plex panel were used (Bio-Rad Laboratories), which included 27 cytokines [IL-1 $\beta$, IL-1ra, IL-2, IL-4, IL-5, IL-6, IL-7, IL-8, IL-9, IL-10, IL-12 (p70), IL-13, IL-15, IL17, Eotaxin, fibroblast growth factor basic (FGF basic), granulocyte colony-stimulating factor (G-CSF), GM-CSF, interferon- $\gamma$ (IFN- $\gamma)$, interferon-inducible protein-10 (IP-10), monocyte chemoattractant protein-1 (MCP-1), MIP-1 $\alpha$, MIP-1 $\beta$, platelet-derived growth factor bb (PDGF bb), regulated upon activation, normal $\mathrm{T}$ expressed and secreted (RANTES), TNF- $\alpha$ and vascular endothelial growth factor (VEGF)]. The samples were analyzed in duplicate wells. The data was analyzed using Bio-Plex Manager 4.0 software (Bio-Rad Laboratories). The detectable lower concentration of each cytokine was about $2.0 \mathrm{pg} / \mathrm{ml}$.
Statistical Analysis The data are expressed as the mean values \pm S.D. The comparisons of non-treatment $v s$. PG treatment were performed by two-tailed paired $t$-test or Wilcoxon signed-rank test, depending on the data distribution. A $p$ value was considered to be statistically significant at a value of $p<0.05$. Statistical analysis was performed using Excel 2003 software (Microsoft, U.S.A.) with the add-in software Statcel 2. ${ }^{22)}$

\section{RESULTS}

Phenotypic Characteristics of DCs Stimulated by WPG iDCs induced by rhGM-CSF plus rhIL-4 expressed CD86 and HLA-DR, but expressed either no or only trace levels of CD80 and CD83 (Fig. 1A). When iDCs were stimulated by W-PG alone $(100 \mu \mathrm{g} / \mathrm{ml})$, the percentage of CD40 positive cells was significantly lower than that of the iDCs (Fig. 1B). Furthermore, the positive percentage and MFI for CCR 5 of the DCs stimulated by W-PG were lower than that of iDCs (positive percentage $p<0.05$ by paired $t$-test, MFI $p=0.056$ by the Wilcoxon signed-ranks test; Fig. 1B). The expression of CD83 on DCs is a phenotypic characteristic of mDCs. ${ }^{23)}$ However, the DC stimulated by W-PG expressed either no or only low levels of CD83.

Phenotypic Characteristics of DCs Stimulated by MIX or MIX+W-PG Next, the effects of a combination of MIX plus W-PG were examined. iDCs were stimulated by MIX or a combination of MIX + W-PG $(100 \mu \mathrm{g} / \mathrm{ml})$ for $2 \mathrm{~d}$. The DCs stimulated by MIX expressed high levels of co-stimulatory molecules and CD83 and thus showed the characteristics of mDCs (Fig. 2A). The phenotype of DCs stimulated by MIX + W-PG was similar to DCs stimulated by MIX alone (Fig. 2A). Although the iDCs expressed low levels of surface CCR7 (Fig. 1A), the DCs stimulated by MIX expressed high levels of surface CCR7 (Fig. 2). The combination of MIX+ W-PG increased the positive percentage and MFI of CCR7 in comparison to MIX alone, while salmon PG $(100 \mu \mathrm{g} / \mathrm{ml})$ showed no effect (Fig. 2B). However, this up-regulation of surface CCR7 expression was not observed in the combination with a lower concentration of W-PG $(1,10 \mu \mathrm{g} / \mathrm{ml}$; data not shown). Therefore, the effects of MIX $+\mathrm{W}-\mathrm{PG}(100 \mu \mathrm{g} /$ $\mathrm{ml}$ ) on $\mathrm{mDCs}$ were analyzed in subsequent experiments.

Effect of W-PG on Intracellular CCR7 Expression of DCs Intracellular CCR7 expression was analyzed to investigate the effect of $\mathrm{W}-\mathrm{PG}$ on total $\mathrm{CCR} 7$ production. Although the surface CCR7 expression of iDCs and DCs stimulated by W-PG alone was a trace level (Fig. 1A), their intracellular CCR7 expression was observed to some extent (Fig. 3). The MIX stimulation significantly increased the intracellular CCR7 expression. In accordance with the up-regulation of surface CCR7 expression, the combination of MIX $+\mathrm{W}$ PG led to the up-regulation of intracellular CCR7 expression in comparison to MIX alone (Fig. 3).

Functional Characteristics of DCs Stimulated by MIX or MIX+W-PG Although the chemotactic responsiveness of iDCs to MIP-3 $\beta$ was less than $2 \%$, the MIX-stimulated DCs had high chemotaxis in response to MIP-3 $\beta$ (Fig. 4). In line with the up-regulation of surface CCR7 expression, the chemotactic responsiveness of DCs stimulated by MIX $+\mathrm{W}$ PG $(100 \mu \mathrm{g} / \mathrm{ml})$ was higher than that of MIX-stimulated DCs (Fig. 4). On the other hand, the chemotactic responsiveness 


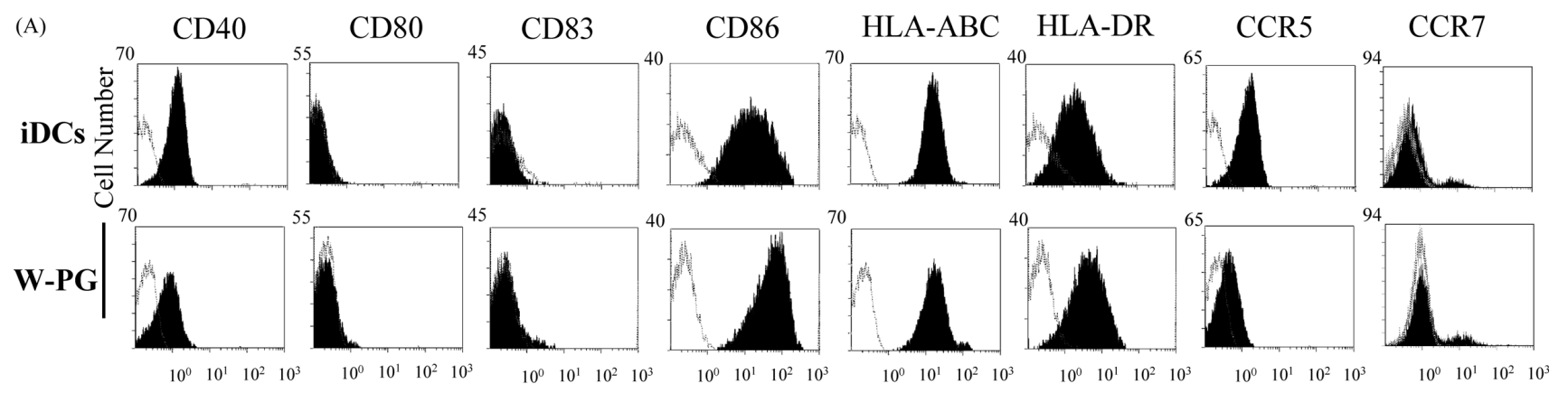

Fluorescence Intensity
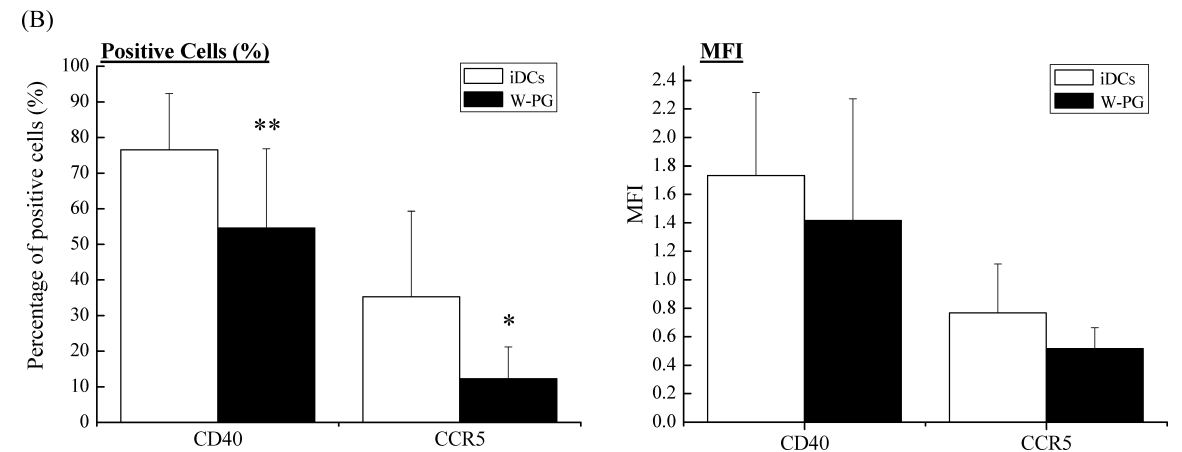

Fig. 1. Cell Surface Antigen Expression on DCs Stimulated by PGs

(A) The immature dendritic cells (iDCs) induced by rhGM-CSF and rhIL-4 were stimulated for $4 \mathrm{~d}$ by proteoglycan extracted from the nasal septum cartilage of whale (W-PG). The iDCs and DCs stimulated by W-PG were stained with the specific mAbs (filled black histogram) or isotype control mAbs (broken line) and then analyzed by flow cytometry. (B) The percent positive cells (\%) and mean fluorescence intensity (MFI) for CD40 and CCR5 of iDCs or DCs stimulated by W-PG are expressed as the mean \pm S.D. of six different individuals. $*$ and $* *$ indicate $p<0.05$ and $p<0.01$ by paired $t$-test, respectively.

(A)
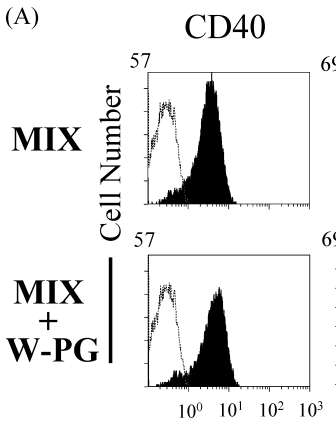

CD80
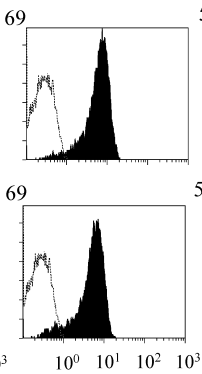

CD83
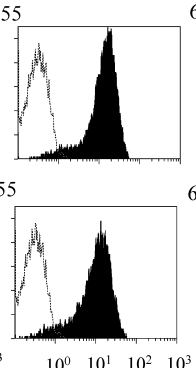

CD86
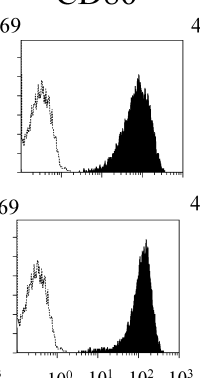
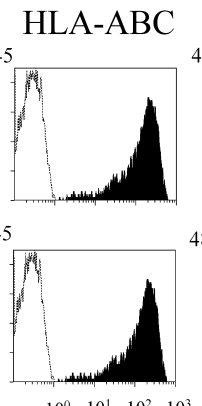
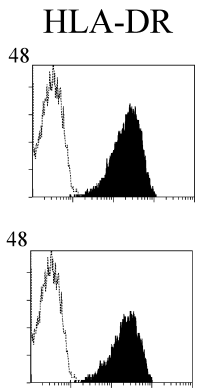

$\begin{array}{lllll}10^{0} & 10^{1} & 10^{2} & 10^{3}\end{array}$
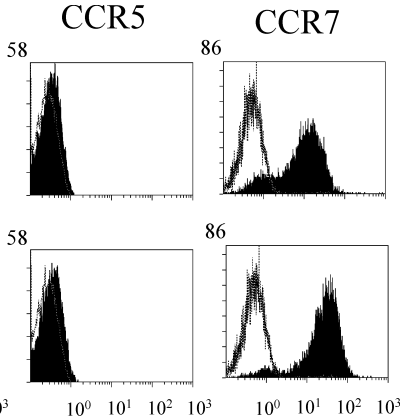

$\begin{array}{llll}10^{0} & 10^{1} & 10^{2} & 10^{3}\end{array}$

Fluorescence Intensity
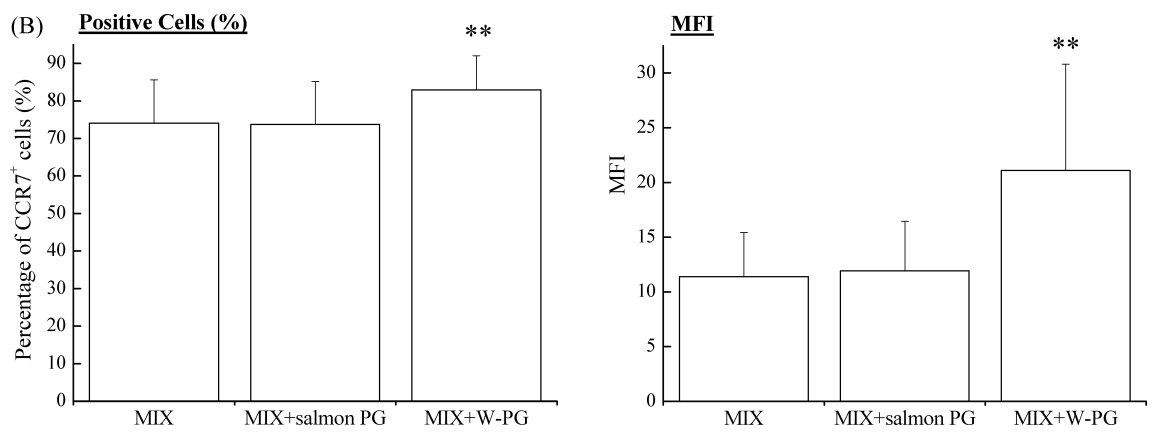

Fig. 2. Cell Surface Antigen Expression on DCs Stimulated by MIX or a Combination of MIX + W-PG

(A) The iDCs were stimulated by MIX ( $10 \mathrm{ng} / \mathrm{ml}$ rhTNF- $\alpha, 10 \mathrm{ng} / \mathrm{ml}$ rhIL- $1 \beta, 10 \mathrm{ng} / \mathrm{ml}$ rhIL- 6 and $1 \mu \mathrm{g} / \mathrm{ml} \mathrm{PGE}$ ) or MIX+W-PG $(100 \mu \mathrm{g} / \mathrm{ml})$ for $2 \mathrm{~d}$. DCs stimulated by MIX or MIX+W-PG were stained with the specific mAbs (filled black histogram) or isotype control mAbs (broken line) and then analyzed by flow cytometry. (B) The percent positive cells and MFI for CCR7 of DCs stimulated by MIX or a combination of MIX plus W-PG or salmon PG (100 $\mu \mathrm{g} / \mathrm{ml}$, respectively) are expressed as the mean \pm SD of six different individuals. ** indicates $p<0.01$ by paired $t$-test. 

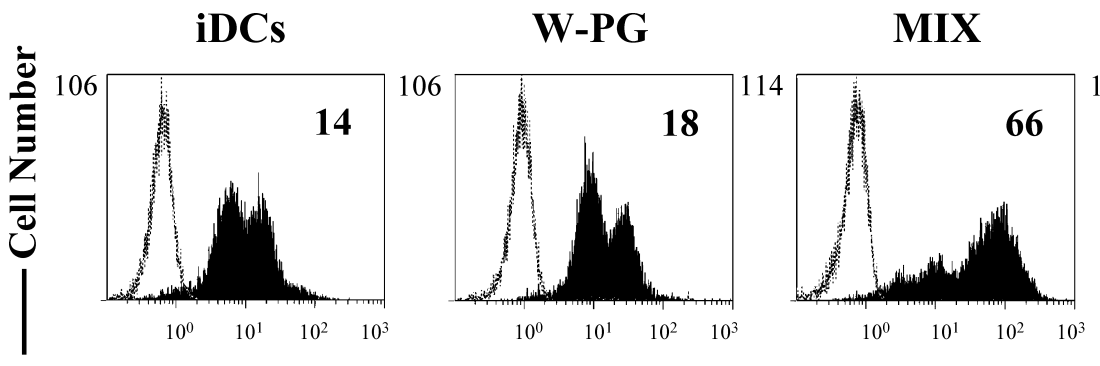

MIX+W-PG

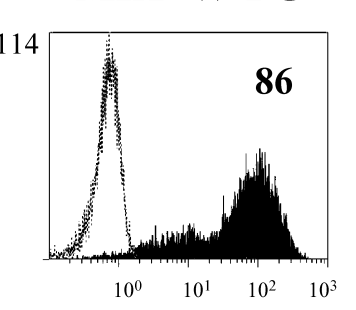

Fluorescence Intensity

Fig. 3. Intracellular Expression of CCR7

The iDCs were stimulated by W-PG alone $(100 \mu \mathrm{g} / \mathrm{ml})$ for $4 \mathrm{~d}$ or MIX or MIX+W-PG $(100 \mu \mathrm{g} / \mathrm{ml})$ for $2 \mathrm{~d}$. After 2 or $4 \mathrm{~d}$ of culture, the DCs were harvested and permeabilized. After permeabilization, the cells were treated for CCR7 staining (filled black histogram) or stained with FITC-conjugated goat anti-mouse IgM antibodies (broken line) as described in the Materials and Methods. The data are representative of three different individuals. The values inserted into histograms indicate MFI.

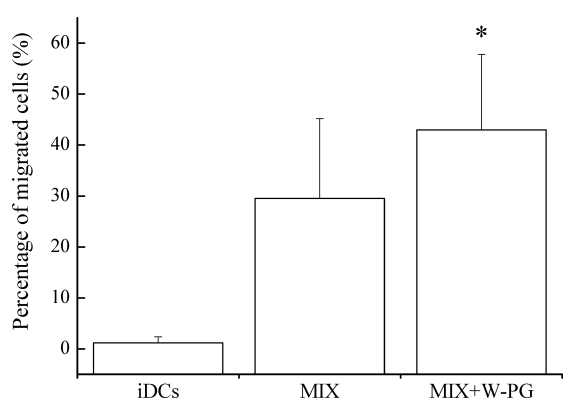

Fig. 4. Chemotactic Responsiveness of DCs to MIP-3 $\beta$

The iDCs were stimulated by MIX or MIX + W-PG $(100 \mu \mathrm{g} / \mathrm{ml})$ for $2 \mathrm{~d}$. Each DC was loaded into the upper chamber. MIP-3 $\beta(100 \mathrm{ng} / \mathrm{ml})$ used as a chemoattractant was added to the lower chamber. After $2 \mathrm{~h}$ incubation, the cells that had migrated to the lower chamber were harvested and counted by flow cytometry. The results are shown as the percentage of migrated cells. The data are mean \pm S.D. of two (for iDCs) or five (for MIX and MIX + W-PG) different individuals. * indicates $p<0.05$ by paired $t$-test.

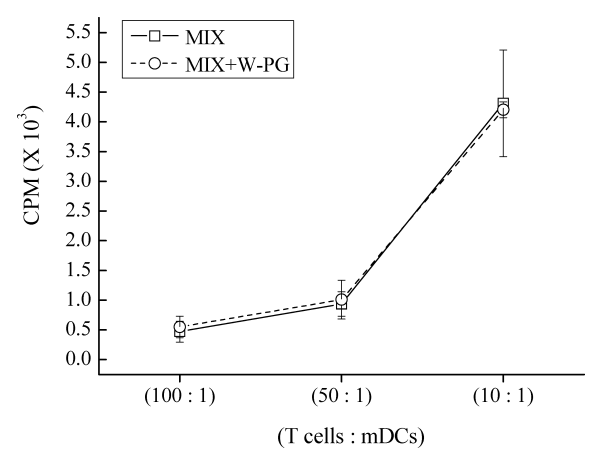

Fig. 5. Stimulating Ability of DCs on Allogeneic T Cell Proliferation

The iDCs were stimulated by MIX or MIX+W-PG $(100 \mu \mathrm{g} / \mathrm{ml})$ for $2 \mathrm{~d}$. Each DC was co-cultured with allogeneic $\mathrm{CD} 4^{+} \mathrm{T}$ cells $\left(1.0 \times 10^{5}\right.$ cells $)$ for $3 \mathrm{~d}$. The cells were further incubated for $20 \mathrm{~h}$ in the presence of $\left[{ }^{3} \mathrm{H}\right]$-thymidine. $\mathrm{CD} 4^{+} \mathrm{T}$ cell proliferation was determined by the incorporation of $\left[{ }^{3} \mathrm{H}\right]$-thymidine. The incorporation of $\left[{ }^{3} \mathrm{H}\right]$ thymidine by only $\mathrm{CD}^{+} \mathrm{T}$ cells was less than $90 \mathrm{cpm}$. The data are the mean \pm S.D. of four different individuals.

of DCs stimulated by a combination of MIX + salmon PG was the same level as that of MIX-stimulated DCs. ${ }^{13)}$

mDCs can stimulate the proliferation of allogeneic leukocytes and this ability is often used as a surrogate marker of their activation. Therefore, the ability of the DCs in allogeneic mixed leukocytes reaction was examined. Each DC stimulated allogeneic $\mathrm{CD}^{+} \mathrm{T}$ cells (Fig. 5). However, the addition of W-PG to MIX did not influence the ability of DCs to stimulate allogeneic CD ${ }^{+} \mathrm{T}$ cells.

Cytokine Detected in the Culture Supernatant of DCs The cytokines contained in the culture supernatants of the
DCs stimulated by MIX or MIX+W-PG $(100 \mu \mathrm{g} / \mathrm{ml})$ were measured (Table 1). Since GM-CSF, IL-4, IL-1 $\beta$, IL-6 and TNF- $\alpha$ were added to the culture medium during the processing from monocytes to DCs, a large amount of those cytokines were detected and their concentrations, except TNF$\alpha$, exceeded the detectable upper limits of the cytokines in most individuals. As shown in Table 1, a statistically significant difference was observed in some of the cytokine concentrations contained in the culture supernatants. The addition of W-PG to MIX increased the concentration of IL-15 and G-CSF to 1.17-fold and 1.36-fold, respectively. In contrast to the increase in these cytokines, the addition of W-PG to MIX decreased the concentrations of IL-7, IL-17, MIP-1 $\beta$, Eotaxin, TNF- $\alpha$ and PDGF bb. In MIP- $1 \beta$, TNF- $\alpha$ and PDGF bb, large decreases in the concentrations were observed $(15,28,37 \%$, respectively), while the decreases in the concentrations of IL-7, IL-17 and Eotaxin by addition of WPG were relatively low $(7,10,3 \%$, respectively).

\section{DISCUSSION}

Recently, we have reported that salmon PG is not able to induce the maturation of human monocyte-derived DCs. ${ }^{13}$ ) The present study investigated the effects of W-PG on the maturation of human monocyte-derived DCs. However, the stimulation of W-PG also did not promote a sufficient maturation of DCs (Fig. 1), though the combination of MIX and W-PG, not salmon PG, increased the CCR7 expression and the chemotactic responsiveness to MIP-3 $\beta$ (Figs. 2-4). ${ }^{13)}$ The major difference between salmon PG and W-PG is the species, thus suggesting the existence of differences in the core proteins and composition of GAGs. ${ }^{14)}$ The W-PG contains 4-sulfated unsaturated disaccharide units more than salmon PG. Chondroitin A and B which are structurally 4sulfated, but not $\mathrm{C}$ which is 6-sulfated, can activate monocytes to secrete IL- $1 \beta$ and B cells to proliferate, respectively. ${ }^{24)}$ Furthermore, chondroitin sulfate A promotes the differentiation of DCs derived from human peripheral blood monocytes with up-regulation of co-stimulatory molecules and HLA-DR expression. ${ }^{6}$ ) The major carbohydrate component of the fruit body of Agaricus blazei is $\beta$-glucan (mainly $1,6-\beta$-glucan $)^{25)}$ and PGs purified from the Agaricus blazei could induce maturation of DCs derived from murine bone marrow. ${ }^{8)}$ These findings indicate that the smaller molecules such as polysaccharides and GAGs may be able to better activate the immune response than enormous molecules such as 
Table 1. Cytokine Concentration Detected in the Culture Supernatants of Each DC

\begin{tabular}{|c|c|c|c|c|c|c|c|}
\hline & \multicolumn{2}{|c|}{ MIX } & \multicolumn{2}{|c|}{$\mathrm{MIX}+\mathrm{W}-\mathrm{PG}$} & \multicolumn{2}{|c|}{ Ratio of concentration } & \\
\hline & Mean & S.D. & Mean & S.D. & Mean & S.D. & \\
\hline IL- $1 \beta$ & $>2300$ & & $>2300$ & & & & \\
\hline IL-1ra & $>10000$ & & $>10000$ & & & & \\
\hline IL-2 & 4.83 & 0.62 & 4.65 & 0.44 & 0.97 & 0.09 & n.s. \\
\hline IL-4 & $>2200$ & & $>3100$ & & & & \\
\hline IL-5 & $<2.0$ & & $<2.0$ & & & & \\
\hline IL-6 & $>22100$ & & $>22100$ & & & & \\
\hline IL-7 & 3.28 & 0.40 & 3.04 & 0.39 & 0.93 & 0.08 & $*$ \\
\hline IL-8 & $>1700$ & & $>1700$ & & & & \\
\hline IL-9 & 51.34 & 6.51 & 49.69 & 3.39 & 0.98 & 0.09 & n.s. \\
\hline IL-10 & 23.92 & 3.83 & 22.84 & 3.61 & 0.96 & 0.08 & n.s. \\
\hline IL-12 & 62.80 & 9.69 & 61.01 & 7.56 & 0.98 & 0.07 & n.s. \\
\hline IL-13 & 7.42 & 4.05 & 7.19 & 4.32 & 0.96 & 0.10 & n.s. \\
\hline IL-15 & 8.78 & 0.99 & 10.24 & 1.21 & 1.17 & 0.14 & $*$ \\
\hline IL-17 & 11.02 & 2.20 & 9.87 & 1.95 & 0.90 & 0.14 & $* *$ \\
\hline Eotaxin & 47.32 & 4.20 & 45.71 & 2.69 & 0.97 & 0.04 & $*$ \\
\hline FGF basic & 26.74 & 2.31 & 26.73 & 2.27 & 1.00 & 0.06 & n.s. \\
\hline G-CSF & 80.50 & 93.60 & 106.28 & 110.61 & 1.36 & 0.23 & $* *$ \\
\hline GM-CSF & $>4300$ & & $>4300$ & & & & \\
\hline IFN- $\gamma$ & 390.48 & 34.74 & 375.42 & 16.66 & 0.97 & 0.06 & n.s. \\
\hline IP-10 & 198.97 & 141.19 & 188.72 & 104.68 & 1.10 & 0.43 & n.s. \\
\hline MCP-1 & $>430$ & & $>350$ & & & & \\
\hline MIP-1 $\alpha$ & 29.23 & 21.70 & 26.16 & 12.14 & 1.02 & 0.24 & n.s. \\
\hline MIP-1 $\beta$ & 155.15 & 123.01 & 117.49 & 71.52 & 0.85 & 0.19 & \# \\
\hline PDGF bb & 1513.26 & 1658.24 & 823.21 & 678.01 & 0.63 & 0.16 & \# \\
\hline RANTES & 43.67 & 26.55 & 39.58 & 20.97 & 0.98 & 0.23 & n.s. \\
\hline TNF- $\alpha$ & 3368.05 & 1016.10 & 2343.89 & 771.84 & 0.72 & 0.19 & $* *$ \\
\hline VEGF & 6739.31 & 2679.85 & 6214.78 & 2792.00 & 0.94 & 0.19 & n.s. \\
\hline
\end{tabular}

The cytokine concentration unit is $\mathrm{pg} / \mathrm{ml}$. The data of nine different individuals are shown. When the cytokine concentrations contained in each culture supernatant exceeded the detectable upper limits in some individuals, the detected lowest concentration is shown (in brief, $>$ the detected lowest concentration). The concentration of IL-5 less than $2.0 \mathrm{pg} / \mathrm{ml}$. The ratio of concentration was obtained by calculating the value of MIX+W-PG/MIX in each individual. $*$ and $* *$ indicate $p<0.05$ and $p<0.01$ by paired $t$-test, respectively. \# indicates $p<0.05$ according to the two-tailed Wilcoxon signed-ranks test. n.s. means not significant.

PG in the maturation of DCs. Therefore, although further studies are needed to determine the important compartments of the PG (i.e. core protein or GAGs), it is thought that the difference in the composition of GAGs leads to the different effects of PG on the human monocyte-derived DCs.

Not only chemotaxis but also degradation of the extracellular matrix by MMPs is required for the migration of DCs. ${ }^{26-29)}$ However, no large difference in the total MMP-9 activity was observed between the W-PG treatment and no treatment (data not shown). In this study, an attractive finding was the fact that W-PG had an effect on the CCR7 expression of DCs and their chemotactic responsiveness in combination with MIX without modulating the MMP-9 and T lymphocyte proliferation activities (Figs. 2, 4, 5). Del Prete et al. reported that a low concentration of leukotriene $\mathrm{B}_{4}$ enhances both CCR7 membrane expression and the chemotactic responsiveness of mouse iDCs without affecting total CCR7 expression. ${ }^{30)}$ Therefore, the localization of CCR7 onto the cell surface is important for its functions. The effect of W-PG treatment is probably due to the increase of CCR7 protein, and then results in their localization onto the cell surface.

MIX is known to be a gold standard stimulant for the ex vivo differentiation of DCs. These results therefore showed that glyco-proteins such as W-PG may be an additive reagent for processing more functional DCs. To identify this specific role of W-PG we assayed multiple cytokines and chemokines since the cytokines released from DCs are known to enable not only paracrine activations to lymphocytes but also autocrine activations to themselves. ${ }^{31,32)}$ A significant differ- ence was observed in the secretion of certain cytokines between the stimulation with MIX and MIX+W-PG (Table 1). iDCs express a unique repertoire of inflammatory chemokine receptors such as CCR2, CCR3, and CCR5 ${ }^{4,19)}$ The CCR5 expression on iDCs decreased after stimulation by W-PG alone (Fig. 1B). Furthermore, the MIP-1 $\beta$ (ligand for CCR5) and Eotaxin (ligand for CCR3) ${ }^{4,33)}$ were both significantly down-regulated by the addition of W-PG (Table 1). These results may suggest that W-PG helps DCs migrate from the inflammatory site to the peripheral lymphatic vessels. PDGF $\mathrm{bb}$ affects the regulation of the growth and migration in many cell types including monocytes. ${ }^{34)}$ A significant decrease in PDGF bb by W-PG was observed (Table 1). Therefore, the suppression of PDGF bb may be involved with the up-regulation of the CCR7 expression. However, the anti-human PDGF bb neutralizing antibody did not affect the surface CCR7 expression in our preliminary examinations (data not shown). There is also a possibility that W-PG enhances the responsiveness of DCs to TNF- $\alpha$, because the remaining amount of TNF- $\alpha$ contained in the culture supernatants was significantly lower in the MIX+W-PG compared with MIX (Table 1). These results demonstrated that the addition of WPG could change the cytokine and chemokine environment although its key factor still remains unclear. Furthermore, previous studies have demonstrated that the presence of $\mathrm{PGE}_{2}$ during maturation confers a functional chemotactic responsiveness to CCL19 and CCL21 via CCR7 in the human monocyte-derived mDCs. ${ }^{35-37)}$ Therefore, an effect of W-PG on the signal transduction of $\mathrm{PGE}_{2}$ contained in MIX cannot 
be ruled out.

In conclusion, the current study indicated that PG extracted from the nasal septum cartilage of whale modulates the migration of DCs via the up-regulation of CCR7 expression. CCR7 plays an important role in the immune response, because it is involved in not only the chemotaxis but also other functions such as the cytoarchitecture, and the survival and maturation of the DCs. ${ }^{38)}$ Interestingly, the effects of PG depend on the types of species because PG from nasal cartilage of salmon head has no effect on the chemotaxis of human monocyte-derived DCs. ${ }^{13)}$ Further understanding of its mechanism and studies using human PGs will provide insight into the immune response, such as the migration of immune cells including DCs in vitro and in vivo.

Acknowledgments We thank the Aomori Red Cross Blood Center for providing the buffy coats. This study was supported by the City Area Program for Promotion of Science and Technology in Regional Areas from the Japanese Ministry of Education, Culture, Sports, Science and Technology (2007-2009). This study was also supported by Japanese grants (KAKENHI) as follows; Grants-in Aid for Young Scientists (B), No. 20790874 KT and Grants-in Aid for Scientific Research (B), No. 18390327 IK, and was supported by a Grant for Priority Research on Issues of Great Urgency Designated by the President of Hirosaki University (2007) and a Grant for Hirosaki University Institutional Research (2008-2009).

\section{REFERENCES}

1) Banchereau J., Steinman R. M., Nature (London), 392, 245-252 (1998).

2) Banchereau J., Briere F., Caux C., Davoust J., Lebecque S., Liu Y. J., Pulendran B., Palucka K., Annu. Rev. Immunol., 18, 767-811 (2000).

3) O’Neill D. W., Adams S., Bhardwaj N., Blood, 104, 2235-2246 (2004).

4) Sozzani S., Cytokine Growth Factor Rev., 16, 581-592 (2005).

5) Termeer C., Sleeman J. P., Simon J. C., Trends Immunol., 24, 112 114 (2003)

6) Yang R., Yan Z., Chen F., Hansson G. K., Kiessling R., Scand. J. Immunol., 55, 2-13 (2002).

7) Kim G. Y., Oh W. K., Shin B. C., Shin Y. I., Park Y. C., Ahn S. C., Lee J. D., Bae Y. S., Kwak J. Y., Park Y. M., FEBS Lett., 576, 391-400 (2004).

8) Kim G. Y., Lee M. Y., Lee H. J., Moon D. O., Lee C. M., Jin C. Y., Choi Y. H., Jeong Y. K., Chung K. T., Lee J. Y., Choi I. H., Park Y. M., Int. Immunopharmacol., 5, 1523-1532 (2005).

9) Park S. K., Kim G. Y., Lim J. Y., Kwak J. Y., Bae Y. S., Lee J. D., Oh Y. H., Ahn S. C., Park Y. M., Biochem. Biophys. Res. Commun., 312, $449-458$ (2003).

10) Majima M., Takagaki K., Sudo S., Yoshihara S., Kudo Y., Yamagishi
S., "New Developments in Glycomedicine," ed. by Endo M., Excepta Medica International Congress Series, No.1223, Elsevier Science, Amsterdam, 2001.

11) Sashinami H., Takagaki K., Nakane A., Biochem. Biophys. Res. Commun., 351, 1005-1010 (2006).

12) Kashiwakura I., Takahashi K., Takagaki K., Glycoconj. J., 24, 251258 (2007).

13) Yoshino H., Takahashi K., Monzen S., Kashiwakura I., Biol. Pharm. Bull., 33, 311-315 (2010).

14) Kashiwakura I., Takahashi K., Monzen S., Nakamura T., Takagaki K., Life Sci., 82, 1023-1031 (2008).

15) Oike Y., Kimata K., Shinomura T., Nakazawa K., Suzuki S., Biochem. J., 191, 193-207 (1980).

16) Yoshino H., Takahashi K., Monzen S., Kashiwakura I., J. Radiat. Res. (Tokyo), 49, 293-303 (2008).

17) Repnik U., Knezevic M., Jeras M., J. Immunol. Methods, 278, 283 292 (2003).

18) Sallusto F., Lanzavecchia A., J. Exp. Med., 179, 1109-1118 (1994).

19) Sato K., Kawasaki H., Nagayama H., Enomoto M., Morimoto C., Tadokoro K., Juji T., Takahashi T. A., J. Immunol., 164, 2285-2295 (2000).

20) Takei M., Umeyama A., Arihara S., Eur. J. Pharmacol., 537, 190199 (2006).

21) Carson R. T., Vignali, D. A., J. Immunol. Methods, 227, 41-52 (1999).

22) Yanai H., "Statcel-The Useful Add-in Software Forms on Excel," 2nd ed., OMS, Tokyo, 2006.

23) Zhou L. J., Tedder T. F., J. Immunol., 154, 3821-3835 (1995).

24) Rachmilewitz J., Tykocinski M. L., Blood, 92, 223-229 (1998).

25) Ohno N., Furukawa M., Miura N. N., Adachi Y., Motoi M., Yadomae T., Biol. Pharm. Bull., 24, 820-828 (2001).

26) Osman M., Tortorella M., Londei M., Quarationo, S., Immunology, 105, 73-82 (2002).

27) Kouwenhoven M., Ozenci V., Tjernlund A., Pashenkov M., Homman M., Press R., Link H., J. Neuroimmunol., 126, 161-171 (2002).

28) Kobayashi Y., Matsumoto M., Kotani M., Makino T., J. Immunol., 163, 5989-5993 (1999).

29) Ichiyasu H., McCormack J. M., McCarthy K. M., Dombkowski D., Preffer F. I., Schneeberger E. E., Am. J. Respir. Cell Mol. Biol., 30, $761-770$ (2004).

30) Del Prete A., Shao W.H., Mitola S., Santoro G., Sozzani S., Haribabu B., Blood, 109, 626-631 (2007).

31) Frucht D. M., Fukao T., Bogdan C., Schindler H., O’Shea J. J., Koyasu S., Trends Immunol., 22, 556-560 (2001).

32) Blanco P., Palucka A. K., Pascual V., Banchereau J., Cytokine Growth Factor Rev., 19, 41-52 (2008).

33) Rollins B. J., Blood, 90, 909-928 (1997).

34) Siegbehn A., Hammacher A., Westermark B., Heldin C. H., J. Clin. Invest., 85, 916-920 (1990).

35) Scandella E., Men Y., Gillessen S., Förster R., Groettrup M., Blood, 100, 1353-1361 (2002).

36) Scandella E., Men Y., Legler D. F., Gillessen S., Prikler L., Ludewig B., Groettrup M., Blood, 103, 1595-1601 (2004).

37) Legler D. F., Krause P., Scandella E., Singer E., Groettrup M., J. Immunol., 176, 966-973 (2006).

38) Sánchez-Sánchez N., Riol-Blanco L., Rodríguez-Fernández J. L., J. Immunol., 176, 5153—5159 (2006). 